\title{
LASER BASED SURFACE PROCESSING OF ENGINEERING MATERIALS - STATE OF ART
}

\author{
Palani I.A. , Vasa N.J. ${ }^{2}$, Kanmani Subbu S. ${ }^{3}$, Ramkumar J. ${ }^{4}$, Singaperumal M. ${ }^{5}$ \\ ${ }^{1}$ PEIL, Department of Mechanical Engineering, Indian Institute of Technology Madras, Chennai, India \\ ${ }^{2,5}$ Engineering Design Department, Indian Institute of Technology Madras, Chennai, India \\ ${ }^{3,4}$ Manufacturing Engineering Section, Department of Mechanical Engineering, \\ Indian Institute of Technology, Kanpur, India \\ E-mail : palaniia@gmail.com
}

\begin{abstract}
An overview is given on the state of art of the laser based surface processing of engineering materials. In major engineering application surface of the material plays a major role. since the surface has the direct contact with the environment as well as the load. The different parameters such as hardness, toughness, wear resistance etc on the surface has to be enhanced based on the application, hence there is a need to go for surface processing. Conventional surface processing has its own limitations toward recently developed materials and other functional aspects. These aspects can be overcome by laser based surface processing. In this paper an attempt has been made to investigate the principal and effect of laser interaction on materials such as heating, surface melting, vaporization, plasma formation, ablation etc. The different types of laser based surface processing of engineering materials based on their properties, Processing parameters and functional aspects which is considered for different application has been discussed.
\end{abstract}

Key words: Surface Engineering, Laser Based Surface Processing, Engineering Materials

\section{INTRODUCTION}

In the recent technological advancement there is a rapid usage of engineering materials ranging from automotive, aerospace, missile, power, electronic, biomedical, textile, MEMS (Micro Electro Mechanical Systems), chemical, steel, power, cement, machine tools, construction industries etc. The engineering materials basically metals, ceramics, polymers, and include composites in all these materials the surface of the bulk place a major role. Hence the surface of the materials should have appropriate properties based on the application such as high wear resistance, corrosion resistance, high hardness, high toughness etc. The surface engineering has become a core topic which needs an urgent attention for investigation. Surface engineering is the design and modification of the surface or the substrate that provides cost effective and performance enhancement, there are different types of surface modification processes available for different applications but they have their own limitations such as surface processing of intricate shapes, control of process parameters and compatibility to the recently developed materials etc, these limitations are overcome by using laser.

Lasers have become an attractive tool for surface engineering of materials. In 1970s lasers were used for cutting, welding and drilling and marking. Recently lasers are being used for surface related applications such as heat treatment, cladding, alloying, and thin film development. In this paper the principle of laser interaction on engineering materials and the effect of laser interaction and the processing parameters has been investigated, the different types of laser based surface processing such as laser hardening, laser alloying, laser annealing etc for different applications has been discussed in detail.

\section{LASER INTERACTION ON ENGINEERING MATERIALS}

The study on the extent of laser interaction on the material primarily depends on the electromagnetic radiation and the thermo physical properties of the materials. The laser parameters includes intensity, wavelength, spatial and temporal coherence, angle of incidence, polarization, illumination time etc whereas the material parameters include absorbtivity, thermal conductivity, specific heat, density, latent heat etc [1] .

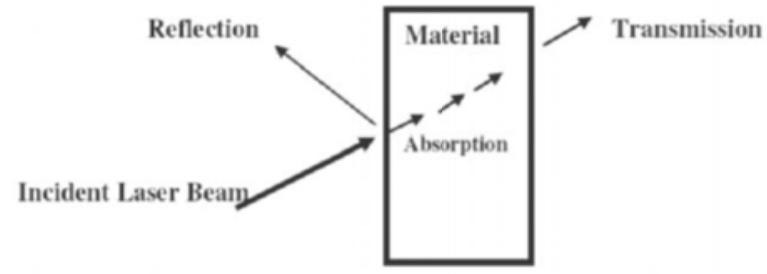

Fig. 1. Laser interaction materials

Laser radiations is an electromagnetic waves, which are associated with electric $(\mathrm{E})$ and magnetic field vector $H$. Absorption of light can be explained as the interaction of the electromagnetic radiation with electrons of the material. Electromagnetic radiations can interact only with electrons of the atoms of the material because it has much heavier nuclei. When the electromagnetic radiation passes over the electron it exerts a force. The force exerted by the electromagnetic radiation of the electron can be expressed as [2-4] 


$$
F=e E+e\left(\frac{v}{c} \times H\right)
$$

Where $\mathrm{n}$ is the velocity of electron and $\mathrm{c}$ is the speed of light. If the electric and magnetic field carry the same amount of energy, the contribution of magnetic field to the force is smaller than that of the electric field by the order of $\mathrm{n} / \mathrm{c}$. Hence, the most important parameter in the above equation is $\mathrm{eE}$. The absorbed radiation thus results in the excess energy of the charged particles such as kinetic energy of the free electrons, localized primary excitation energy of the bound electrons etc. Eventually, the degradation of the ordered and localized primary excitation energy through various steps leads to the generation of heat. Hence the absorption processes is sometimes referred to as the secondary source of energy inside the material The absorption of laser radiation in the material is generally expressed in terms of Beer Lamberts law $[5,6]$

$$
I=(1-R) \times I_{0} \exp (-\alpha \times x)
$$

Where 10 is the incident intensity, I is the intensity at depth $\mathrm{x}$ and $\alpha$ is the absorption coefficient and $R$ is the reflectivity. The equation [7] can also be expressed in terms of extinction coefficient $k$ and wavelength $(\lambda)$ is

$$
I=I_{0} \exp \left(-\frac{4 \pi \mathrm{kx}}{\lambda}\right)
$$

Where $\lambda$ is the wavelength and the extinction coefficient [8] $\mathrm{kis}$

$$
\alpha=\frac{4 \pi \mathbf{k}}{\lambda}
$$

The optical energy absorbed by the material is converted into thermal energy which is responsible for increase in temperature of the material as shown in Fig .2.

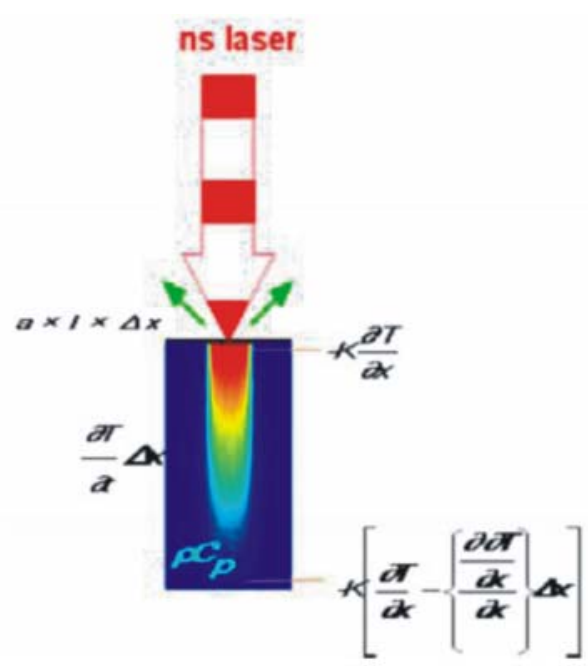

Fig. 2. Laser based Heat flow in the materials

The heat flow due to laser interaction is considered to be one dimensional. In which the amount of heat conducted is the combination of the amount of heat accumulated and the amount of heat induced. Hence the heat balance equation can be derived as [9-11].

$$
\rho C \frac{\partial T}{\partial t}=\alpha \times I+k \frac{\partial^{2} T}{\partial x^{2}}
$$

Where $r$ is the density of the material $(\mathrm{kg} / \mathrm{m} 3)$. $C$ is the specific heat capacity of the material $(\mathrm{J} / \mathrm{kgK}), a$ is the absorption of the material [12], I is the intensity $(\mathrm{W} / \mathrm{m} 2)$ and $\mathrm{K}$ is the thermal conductivity of the material (W/mK).

\section{EFFECT OF LASERS IN SURFACE PROCESSING OF MATERIAL}

The conversion of light energy into heat and its subsequent conduction into the material establishes the temperature distributions in the material. The laser effect in the material includes heating, melting and vaporization of the material. Furthermore, the ionization of vapor during laser irradiation may lead to generation of plasma.

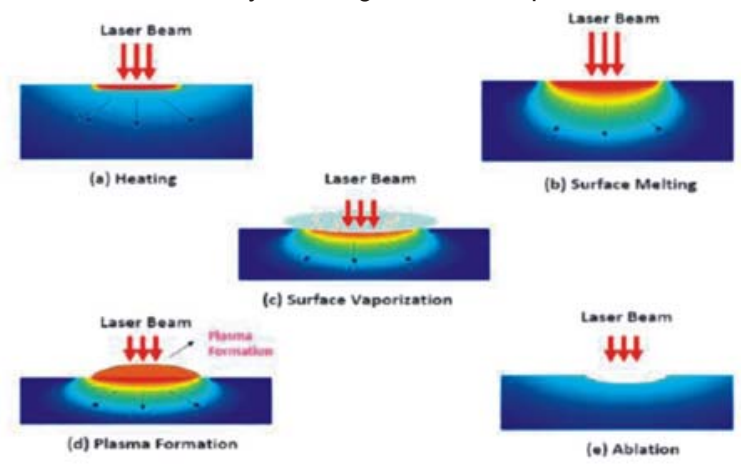

Fig. 3. Effect of laser interaction on material 
In addition to the thermal effects, the laser material interaction may be associated with the photochemical processes such as photoablation of the material [13]. The various effect of laser interaction are explained in Fig. 3.

When a laser beam of intensity 10 is irradiated on the surface of the material and it results in the excitation of free electrons in metals, vibrations in insulators and both in semiconductors [14-16] The excitation energy is directly converted into heat (time duration in the range $10-13 \mathrm{~s}$ for metals, $10-12$ to $10-6 \mathrm{~s}$ for nonmetals) $[17,18]$. This is followed by various heat transfer processes such as conduction into the materials, convection, and radiation from the surface. The generation of heat on the surface and its conduction into the material establishes the temperature distribution in the material depending on the thermo physical properties of the material and the laser parameters [19-21] if the incident laser intensity is sufficiently high it results in phase transformation such as surface melting and evaporation. The depth of melting is the major parameter which has to be analyzed during laser irradiation. The depth cannot be increase to infinitely large values with increasing laser power density and pulse time because the location of the melting point in the temperature to the depth is limited by the maximum achievable surface temperature. Once the surface temperature reaches the boiling point, the depth of melting reaches the maximum value.

Further increase in the laser power density or the laser pulse time causes evaporation of the material removal from the surface without further increase in the depth of melting. As when the material is irradiated still above the melting point it may result in surface evaporation. The interaction between the resulting vapor and the incident laser beam become important in determining the overall effect of the laser irradiation on the material. One of the most important interactions is the ionization of vapor. The highly ionized vapor is termed as plasma. These generations of plasma can greatly influence with interaction of laser radiation with the material. It is convenient to define the laser power density at which the significant ionization of the vapor results in the formation of plasma takes place $[22,23]$. The plasma is generally considered to form near the evaporating surface of the target and remain confined to this region during laser irradiation with intensities just above the laser power densities. The confinement of the stationary plasma near the evaporating surface is generally referred to as plasma coupling [24]. Plasma coupling plays a major role in transferring the energy to the dense phase. When the laser density is sufficiently large to exceed the boiling point and causing rapid vaporization and the process of material removal by thermal stresses and surface vaporization is referred to as thermal ablation. The laser based surface processing can be classified based on their applications and their compatibility towards surface processing. Lasers Material Processing can be grouped under 2 processes: 1) Applications requiring the delivery of limited and well controlled amounts of energy to the work piece, such as annealing,.2) Applications requiring substantial phase transformations in the work pieces such as heat treatments and cladding. The different types of laser based surface processing are explained in the forecoming a paragraphs.

\section{TYPES OF LASER BASED SURFACE PROCESSING OF ENGINEERING MATERIALS}

\section{A. Laser Hardening}

Laser hardenings, is an autogenous method of producing Wear-resistant patterns on discrete surface regions of components. A shaped laser beam is scanned across the component to heat, but not melts, the surface and then allowing rapid quenching by conduction [25]. In laser surface hardening heat input rate is high then bulk of material remains cold and heated surface will cool rapidly by conduction into the bulk [26]. This provides a hardening on the surface of the material through a solid-state transformation that result in the formation of a highhardness microstructure, i.e. martensite as shown in Fig. 4 . The depth of the hardened zone may be altered by varying the amount of heat input provided to the workpiece [27]

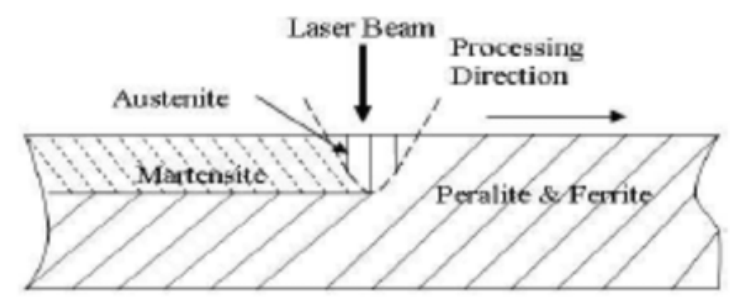

Fig. 4. Laser Hardening of Materials

Laser hardening considers the Composition and geometry in Materials selection and wavelength, power, Power density and Beam interaction time in beam characteristics are the process parameters [25].Laser surface hardening used in automobile industry for axle shaft housings and power steering gear housings. It is also used for selectively hardening typewriter keys, farm implements and parts of hand tools, such as saw teeth and hammer heads [28]. Advantages of laser hardening over conventional methods such as flame hardening and case hardening are the low total heat input gives minimum distortion of the work pieces, heat can be very precisely located, Process is easily automated, Laser beam can be instantly switched on or off and laser beam can be used at several work 
station on a time sharing basis [26, 29].Laser hardening is an effective technique used to improve the tribological properties and also increase the life of components. In this paper they discussed about degree of hardenability and fineness in micro structure obtained depending on the laser processing variables and absorption coefficient coatings employed using High power Co2 lasers, Nd:YAG Lasers and also high power diode lasers using in surface hardening. In this process increases the hardness of the base metal 3-fold increase in hardness was obtained with a maximum case depth of 0.45 and $0.65 \mathrm{~mm}$ at respective laser powers of 1.3 and $1.5 \mathrm{~kW}$. A 2-fold increase in wear resistance was observed for the laserhardened steel [30].Laser hardening of steel or cast-iron components can yield $20 \%$ greater hardness than conventional heattreating. The efficiency of aluminium hardening by radiation from a CW Nd:YAG laser was found to be considerably higher than in the case of a $\mathrm{CW}-\mathrm{CO} 2$ laser [31]. It was found that laser surface hardening of titanium $6 \mathrm{Al}-4 \mathrm{~V}$ alloy which increase the fatigue resistance by using Continuous wave Co2 Laser [32].

\section{B. Laser alloying:}

The laser surface alloying (LSA) is a process in which the desired alloying elements are extraneously added to the melt pool to alter the surface chemical composition as best suited for the part being treated. Such modification of the surface composition to achieve the desired properties can be effected by introducing the alloying elements in gaseous or powder form as a prior coating. In making alloys by Laser, the different process parameters involved are the exposure time, laser power, the thickness of the film put down prior to laser melting, nature of the gaseous ambient during processing. These processes parameters are interrelated and only one variable cannot be freely changed without affecting another (33). Laser alloying is an effective way to increase the surface hardness and wear resistance of materials, including aluminum. The effect of laser alloying on the structure and hardness of silumin AL30 has increased to $180 \mathrm{H}$, which is used to produce engine pistons operating at high temperatures [34]. The laser alloying was done using a pulsed Nd:YAG laser with a generated beam energy of 25-35 J on titanium with bronze as alloying element to increase the hardness and wear resistance for screw propeller and slide bearing . The microhardness of upto $467 \mathrm{HV}$ has been obtained and the wear resistance was considerably increased as compared to the bulk materials [35]. High-quality precipitation hardened stainless steel (17-4PH) with high strength, high antifatigue, excellent corrosion resistance and good weldability widely used to produce steam turbine blades. However, under the impact of high-speed steam and water droplets, the blades are prone to cavitation, 17-
4PH blade's surface was alloyed using a high power $\mathrm{CO} 2$ laser with alloying materials $(2.86 \% \mathrm{Cr}, 3.29 \% \mathrm{Ni}, 0.98 \% \mathrm{Fe}$, $40.24 \% \mathrm{~W}, 51.33 \mathrm{Co}$ ). After laser alloying, the surface layer was denser and the grain refined, while the microhardness of the surface (average 610HV0.2) was about one times higher than that of the substrate material [36]. This laser alloying can also be extended to MEMS application especially in selective doping of semiconductor materials. Laser doping is process in which the dopant materials are made to be diffused onto the substrate as shown in figure. Excimer laser doping technique has been utilized to obtain a heavy impurity doping II-IV semiconductors which are considerably difficult materials to achieve bipolar conductivity and to obtain good ohmic contact on them during device fabrication and the process was successful with good increase in resistivity, carrier mobility and hole concentration [37].
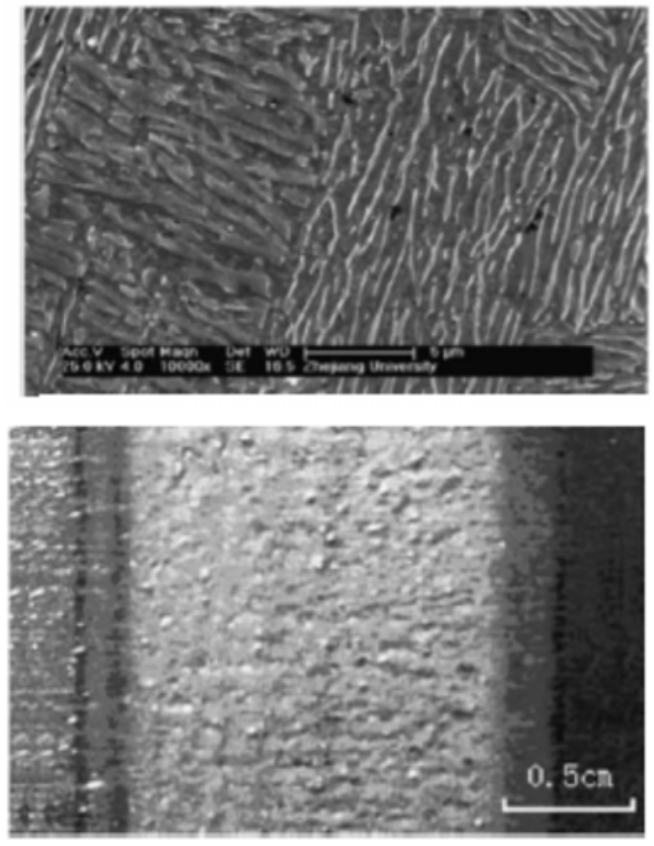

Fig. 5.(a) SEM graph of 17-4PH steel before laser alloying (b) after laser alloying

This laser alloying can also be extended to MEMS application especially in selective doping of semiconductor materials.

\section{B.1. Laserdoping}

Laser doping is process in which the dopant materials are made to be diffused onto the substrate as shown in Fig. 6. 


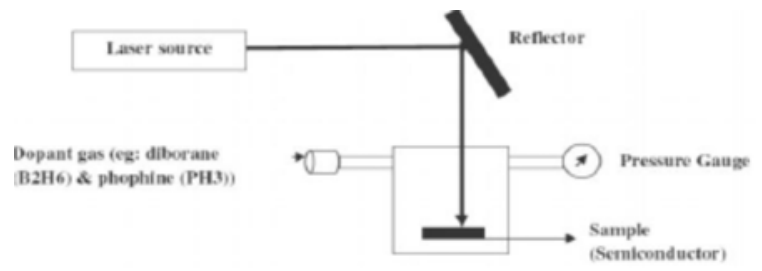

Fig. 6. Laser doping of semiconductors

Excimer laser doping technique has been utilized to obtain a heavy impurity doping II-IV semiconductors which are considerably difficult materials to achieve bipolar conductivity and to obtain good ohmic contact on them during device fabrication and the process was successful with good increase in resistivity, carrier mobility and hole concentration [37].

\section{B.2. Lasercladding}

Laser cladding is a method of depositing material by which a powdered or wire feedstock material is melted and consolidated by use of a laser in order to coat part of a substrate or fabricate a near-net shape part. The powder used in laser cladding is normally of a metallic nature, and is injected into the system by either coaxial or lateral nozzles. The interaction of the metallic powder stream and the laser causes melting to occur, and is known as the melt pool. This is deposited onto a substrate, moving the substrate allows the melt pool to solidify and thus produces a track of solid metal [38]. This is the most common technique; however some processes involve moving the laser/nozzle assembly over a stationary substrate to produce solidified tracks.

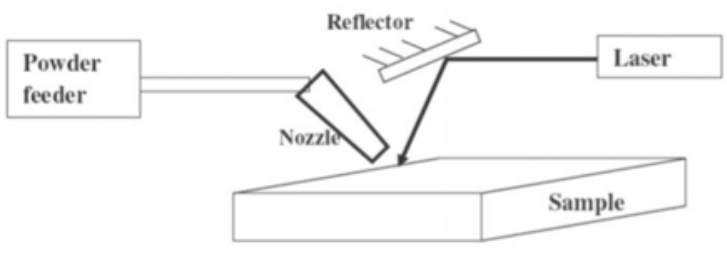

Fig. 7. Laser Cladding of Materials

Hydroxyapatite(HA) a bioceramic coating extensively used for replacing g diseased or injured hard tissues were syntheisied on titanium substrate by laser cladding using cheap calcium carbonate and the process was successful for bioengineering application [39]. Laser cladding was also employed to restore the damaged blades of the Titanium alloy T16242 which is mainly used in lowpressure compressors (LPC) and high pressure compressors (HPC) of aero engines [40].

\section{Laserannealing}

Annealing is a process mainly used to induce recrystallization on the surface of the material to remove the stresses in the highly deformed. This process can also be used to remove the gases trapped in the metal. The annealing is conventionally done in furnace. There are some limitations in furnace annealing such as overlapping of grains structure and cannot be applied to specific areas. These limitations can be overcome by laser annealing technique. Laser annealing is a kinetic process which is mainly used to change the microstructure through diffusion, the laser annealing technique is widely used in MEMS and micro electronics application [41-44]. The laser annealing is mainly used to induce recrystallization on the semiconductor materials to induce the optical and electronic properties. When a short high intensity laser pulse is used to heat the material just below the melting point, the material starts to grow as it cools.

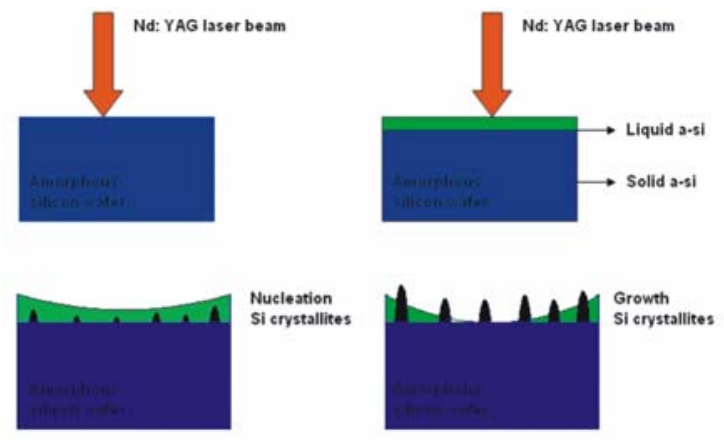

Fig. 8. Stages in laser annealing of Silicon

The SEM photograph shown below can be used to explain between the treated and the untreated monocrystalline silicon. A submicron growth can be clearly seen on the SEM graph of the monocrystalline silicon which has been treated with $\mathrm{Nd}: Y A G$ laser of $350 \mathrm{~mJ} / \mathrm{cm} 2[45]$

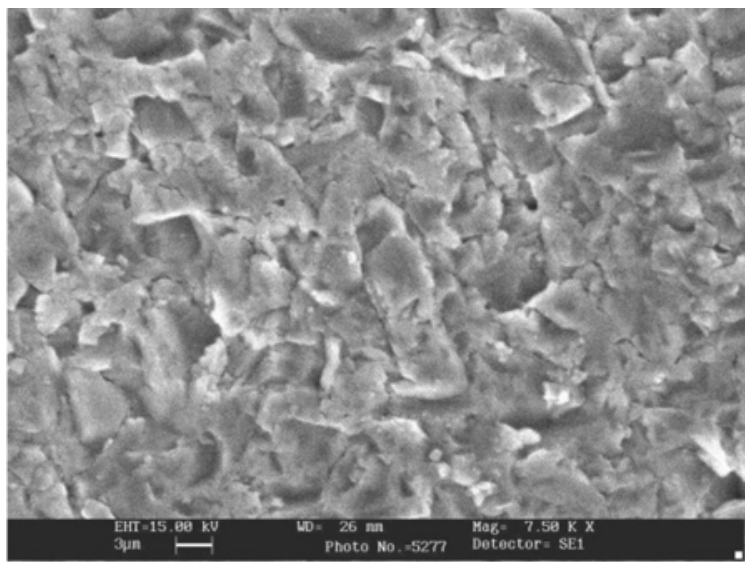

Fig. 9(a). SEM graph of silicon wafer before laser annealing 


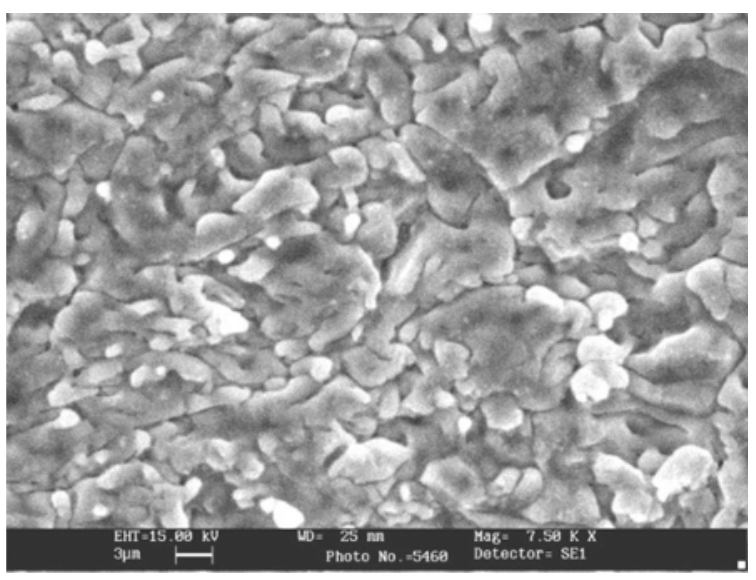

Fig. 9(b). After laser annealing Stages in laser annealing of Silicon

Laser annealing is classified based on the type of Laser used. The pulsed KrF excimer laser annealing on silicon films. The Nano structure on amorphous Silicon surface annealed using nanosecond laser pulse of an excimer laser and the annealing of silicon using $\mathrm{XeCl}$. The surface modification of the silicon wafer by annealing using Transversely Excited Atmospheric (TEA) CO2 laser was also reported [46-49].

\section{Laser shock Processing}

Laser shock peening (LSP) is an innovative surface treatment technique, which is successfully applied to improve fatigue performance of metallic components. After the treatment, the fatigue strength and fatigue life of a metallic material can be increased remarkably owing to the presence of compressive residual stresses in the material. The increase in hardness and yield strength of metallic materials is attributed to high density arrays of dislocations $[50,51]$ and formation of other phases or twins [52] generated by the shock wave.

An LSP process can be used to treat various kinds of metallic components, which include cast irons, aluminum alloys, titanium and its alloys, nickel-based superalloys and so forth. In the aerospace industry, LSP can be used to treat many aerospace products, such as turbine blades and rotor components [53,54], discs, gear shafts[55] and bearing components [56]. Protection of turbine engine components against from foreign object damage (FOD) is a key concern. [57].

A laser pulse that can be adjusted and controlled in real time is a unique advantage of LSP [58]. Regions inaccessible by shot peening (SP), such as small fillets and notches, can still be treated by LSP [59]. A schematic diagram of an LSP process on a metal plate is shown Fig. 10.

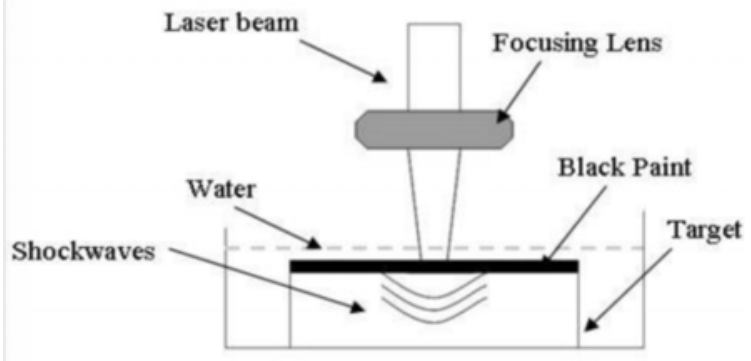

Fig. 10. Laser shock processing of material

When shooting an intense laser beam on to a metal surface for a very short period of time (around $30 \mathrm{~ns}$ ), the heated zone is vaporised to reach temperatures in excess of $10,000^{\circ} \mathrm{C}$ and then is transformed to plasma by ionisation. The plasma continues to absorb the laser energy until the end of the deposition time. The pressure generated by the plasma is transmitted to the material through shock waves. The interaction of the plasma with a metal surface without coating is defined as 'direct ablation', which can achieve a plasma pressure of some tenths of a GPa [60-62]. In order to obtain a high amplitude of shock pressure, an LSP process normally uses a confined mode, in which the metal surface is usually coated with an opaque material such as black paint or aluminium foil, confined by a transparent material such as distilled water or glass against the laser radiation. This type of interaction is called 'confined ablation'. Its had found that, when using the confined mode, ever greater plasma pressures of up to 5-10 GPa could be generated on the metal surface [6365].

The main function of LSP is to introduce surface compressive residual stress or surface strain hardening that can lead to an improvement in the mechanical performance of metallic components such as fatigue and corrosion resistance. LSP can improve fatigue life of aluminium and aluminium alloy by over 30 times and increases the hardness by 80 percent. Micro scale LSP has higher spatial resolution, is more flexible, and is low cost to implement [66].

\section{E. Laserablation}

Laser ablation is the process of removing material from a solid (or occasionally liquid) surface by irradiating it with a laser beam. At low laser flux, the material is heated by the absorbed laser energy and evaporates or sublimates. At high laser flux, the material is typically converted to a plasma. Usually, laser ablation refers to removing material with a pulsed laser, but it is possible to ablate material with a continuous wave laser beam if the laser intensity is high enough. Among conventional polymers that are frequently used in laser ablation studies 
are poly(methyl methacrylate) (PMMA\}, polyimide (PI), poly(tetrafluoroethylene) (PTFE), polyethylene (PE), polycarbonate (PC), and polyamide. An important trend in laser ablation applications involves attempts to create a new polymer with properties specially designed for a desired technique [67]. There is a high amount of research interest in the laser ablation of polymers in specific for the development of micro lens [68-70]. As bio engineering application laser ablation has been used to ablate the dental Tissues [71].

\section{SUMMARY}

The surface of the material acts as a protective barrier in preventing failure to the substrate. To enhance the properties of the surface, laser based surface processing is a versatile tool, it has its own advantages over the conventional laser processing, Hence to investigate the application of laser for processing the surface, the knowledge on the laser interaction on the surface of the materials has been discussed and the different parameters involved in the effect of laser interaction on the materials has been investigated in detail. Finally the different types of laser based surface process has been discussed for diff rent application which includes from automotive, Machine tools to MEMS based applications and it has also been extended to biomedical application too.

\section{REFERENCES}

[1] B.Dahrotre, P.H.kumar, 2007 "Laser fabrication and machining of materials", springer, 2, 65

[2] T.Silfvast,1937,Laser fundamentals, New Delhi, Cambridge university presse,2,9-15.

[3] R.P.Kahre, 2004, Fiber optics and optoelectronics, New Delhi, oxford university, press, 2,18-39.

[4] Breck Hitz et al, 2000, Introduction to Laser Technology, New York,IEEE Press, 2, 7-11.

[5] I.APalani et al, 2007, Study on feasibility of pulsed Nd:YAG laser in annealing of silicon films, National Conference of Research Scholars in Mechanical Engineering, IITK, 187-191.

[6] B.S Yilabs et al, 1998, "Pulsed laser heating of steel surface $-m$ fourier and e;ectron kinetic theory approaches", International committee Heat and Mass Transfer, 25,6, 843-852.

[7] M.Steen, 1991, "Laser Material Processing", Great Britain, Springer, 2, 68.
[8] www.fisk.edu/ aburger/Published03_06/ Introduction/Optical/Extinction_coefficient/extin cti on_coefficient.html - 15k-

[9] C.K.Ong et al, 1988, "Role of thermal conductivity and optical absorption coefficient of amorphous silicon in pulsed laser melting", Mat Sci \& engg , A103,273-276.

[10] I.A.Palani et al, 2008,"Invetsiagtion on Abaltion threshold in annealing of Silicon films by pulsed $\mathrm{Nd}$ :YAG laser" International Conference on Advances in Manufacturing Technology (ICAMT 2008) for Young Engineers, Chennai, India.

[11] M.Kalyon and B.S.Yilnbas, 2003, Laser pulse Heating: formulation of desired temperature at the surface, optics and laser in engineering, $39,109-119$.

[12] http://www.ee.byu.edu/photonics/ tabulatedopticalconstants.phtml

[13] C.Ion, 2005, 'Laser Processing of Engineering Materials-Principal, Procedures and Industrial Application", Burlington, Elsevier.

[14] S.Z.Shuja, B.S.Yilbas, 1998, Pulsative Heating of Surface", International journal of heat and mass transfer, 41, 3899-3918.

[15] Yanbei Chen, et al, 2008,Plastic Penetration During Laser Heating of a Metal Plate, Journal of Materials Processing Technology ,205, 1-3, 9-15.

[16] A.Lietolila and J.F.Gibbons, 1982,"Compuer modeling of the temperature rise and carrier concentration induced in silicon by nanosecond laser pulses"J appl.phys, 53, 4, 3207-3212.

[17] lena Gatskevich et al, 2007,"Modelling laser induced phase transformation in semiconductors", Mathematics and computers in simulation.

[18] P.S. Peercy et al, 1983, "Measurement of melt dynamics in the laser annealed semiconductors", Physica 116B, 558-563.

[19] B.S.Yilbas et al, 1997,"Heat transfer analysis of laser heated surface - conduction limited case", Applied surface science 108, 167-175.

[20] A.F.Hassan et al, 1993, "A general problem of pulse laser heating of a slab" optics \& laser technology, 25, 3, 155-162.

[21] Li Junchang, et al, 1999, "Evaluation of the thermal field development during pulsed laser 
treatments: semi analytical calculation", Surface and coating technology, 115, 87-93.

[22] William Kruer, 2001, The Physics of Laser Plasma Interactions, Westview Press

[23] J.Radziemski, A.Cremers, 1989, Laser-induced Plasmas and Applications, CRC Press.

[24] W. Kruer, 1999, Report on "Laser plasma coupling for high temperature Hohlraums", Lawrence Livermoor National laboratory.

[25] C.Ion, 2005, Laser processing of Engineering Materials, Butterworth, Elsevier, 9, 221.

[26] R. D. Rawlings, W. M. Steen, 1981, Acoustic emission monitoring of surface hardening by laser, Optics and Lasers in Engineering, 2, 3, 173-187.

[27] http://www.mrl.columbia.edu/ntm/level3/ ch05/html//3c05s01.html

[28] http://www.p2pays.org/ref/10/09026.pdf

[29] J.W.Hill, M.J.Lee, I.J.Spalding, 1974, Surface treatments By laser, Optics and Laser Technology, 6, 6, 276-278.

[30] Senthil Selvan.j, Subramanian.k, Nath.A.K, 1999, Effect of Laser surface Hardening on En18 (AISI 5135) steel, Journal of Materials Processing Technology, 91, 29-36.

[31] Ivan B Kovsh et al, 1994, Hardening of aluminium by YAG : Nd laser radiation with an average power of 0.8 kW, Quantum Electro, 24, 141-142.

[32] E. Altus, E. Konstantino, 2001, Optimum laser surface treatment of fatigue damaged Ti-6Al-4V alloy, Materials Science and Engineering $A$, 302, 1, 100-105.

[33] www.answer.com

[34] Yu. B. Kisina et al, 1995, laser surface alloying of selenium Metal science and heat treatment, 37, 1-2.

[35] S. Kac et al, 2007, "Structure and properties of the bronze laser alloyed with titanium", Applied Surface Science, 253, 7895-7898.

[36] Jianhua Yao, Liang Wang et al, 2007, "Surface Laser alloying of $17-4 \mathrm{PH}$ SS steam turbine blades", Optics and Laser technology, 1-6.

[37] Y.Hatanaka, 2001, "Excimer laser doping technique for II-VI semiconductors", Applied surface science, 175-176, 462-467.
[38] Technical report on "Laser surface cladding a literature survey", Leula Tekniska univ, 2000.

[39] D.G. Wange et al, 2008 "In situ synthesis of hydroxyapatite coating by laser cladding" Colloids and Surfaces B: Biointerfaces.

[40] Karl-Hermann Richter et al, 2004, "laser cladding of the titanium alloy Ti6242 to restore damaged blades", Proce of the 23rd Int Congress on Applications of Lasers and Electro-Optics

[41] Sandipm Halder et al, 2006, "Laser annealing of BST thin films with reduce cracking atb an elevated temperatures", Material science and Engineering B.

[42] P.S.Lee et al, 2006, "Multiple -pulse laser thermal annealing for the formatyion of co - silicided junction", IEEE electron device letters , 27,4 .

[43] G.Aygun et al, 2006, "XPS study of pulsed $\mathrm{Nd}$ :YAG laser oxidized Si" Journal of noncrystalline solids, 352, 3135-3139.

[44] Hajime Watakabe et al, 2006, "Electrical properties for poly-Ge films fabricated by pulsed laser annealing", Thin solid films 508, 315-317

[45] I.A.Palani et al, "Investigation on annealing of silicon film by pulsed Nd:YAG laser", 5th Int Conf Precision, Meso,Micro \& Nano(COPEN 07),215-220

[46] H. Azuma et al, 2002, "Pulsed Krf laser annealing of silicon solar cell", Solar Energy Materials \& Solar Cells, 74, 289-294.

[47] D. Klinger et al, 2006, "Nano- structure formed by nanosecond laser annealing on amorphous $\mathrm{Si}$ surface," Mat Sci In Semiconductor Processing, 9, 323-326.

[48] A. Mittiga, L. Fornarini, R. Carluccio "Numerical modelling of Laser induced Phase transition in silicon," Applied Surface Science, 154-155, 2000, 112-11.

[49] K. Milan et al , 2003, "Pulsed TEA CO2 laser surface modification of silicon," Applied Surface Science, 205, 336-342.

[50] G.Banas et al, 1990a, Laser shock induced mechanical and microstructural modification of welded maraging steel, Applied Physics, 67, 2380-4.

[51] G.Banas et al, 1990b, Laser shock hardening of welded maraging steel, Surfa Engg, 67, 280-290. 
[52] J.P.chu, 1999, Laser-shock processing effects on surface microstructure and mechanical properties of low carbon steel, Materials Science and Engineering, A260, 260-8.

[53] S.Mannava and S.J.Ferrigno, 1997, Laser Shock Peening for Gas Turbine Engin Vane Repair. US Patent5675892, GE.

[54] S.Mannava et al, 1997. Laser Shock Peened Gas Turbine Engine Fan Blade Edges. US Patent5591009, GE.

[55] S.J.Ferrigno et al, 2001, Laser Shock Peened Gas Turbine Engine Seal Teeth. US Patent 6200689 , GE.

[56] D.A.Casarcia et al, 1996, Laser Shock Peened Bearings. US Patent 5584 586, GE.

[57] Y.K.Zhang et al, 1997, I"nvestigation of the surface qualities of laser shock-processed zones and the effect on fatigue life of aluminum alloy", Surface and Coatings Technology, 92, 104-9.

[58] S.Mannava, 1998, On the Fly Laser Shock Peening. US Patent 5756 965, GE.

[59] S.Mannava \& W.D Cowie, 1996, Technique to Preventor DivertCracks. USPatent5569018, GE.

[60] Y.sano et al, 1997, "Residual stress improvement in metal surface by underwater laser irradiation", Nuclear Instruments and Methods in Physics Research B, 121, 432-6.

[61] J.E.Masse and G.Barreau, 1995a, "Laser generation of stress waves in metal, Surface and Coatings Technology", 70, 231-4.

[62] J.E. Masse and G.Barreau, 1995b, "Surface modification by laser induced shock waves", Surface Engineering, 11, 131-2.

[63] D.Devaux et al,1997, "Generation of shock waves by laser-matter interaction in confined geometries", Journal De Physique IV, 1(C7), 179-82.
[64] L.Berthe et al,1997, "Shock waves from a waterconfined laser-generated plasma", Journal of Applied Physics, 82, 2826-32.

[65] J.A.Bolger et al,1999, "Shock waves in basalt rock generated with high-powered lasers in a confined geometry, Journal of Applied Physics", 86, 5461-66.

[66] Hwaiyu Gang, 2004, "Mfg Engg Hand book", McGraw-Hill Professional, 34, 34.22.

[67] N.Bityurin, 2005, "Studies on laser ablation of polymers", Annu. Rep. Prog. Chem., Sect. C, 101, 216-247.

[68] T. Efthimiopoulos et al, 2008 "Bubble creation and collapse during excimer laser ablation of weakabsorbing polymers", Applied Surface Science, 254, 5626-5630.

[69] Hirosmi Azuma, 2006, "Surface treatments of polmers by Laser ablation", Technical Report , Toyota Crdl.Inc.

[70] Kris Naessens, "Flexible Fabrication of Microlenses in Polymers with Excimer Laser Ablation".

[71] O.Konorov, 2004, "Laser ablation of dental tissues with picosecond pulses of $1.06 \mathrm{~mm}$ radiation transmitted through a hollow-core photonic-crystal fiber", Applied Optics, 43, 11

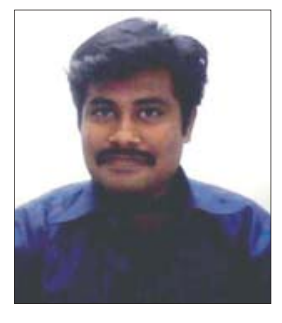

Mr. Palani I. A. is a Research Scholar at the Precision Engineering and Instrumentation Laboratory, Department of Mechanical Engineering, Indian Institute of Technology Madras (I.I.T MADRAS), Chennai. He was awarded the National Doctoral Fellowship 2006-2007(NDF 2006-07) from All India Council for Technical Education (AICTE). His field of research is "Laser based Application in MEMS". 